Draft VERsion March 7, 2018

Preprint typeset using $\mathrm{LAT}_{\mathrm{E}} \mathrm{X}$ style AASTeX6 v. 1.0

\title{
EXCITATION MECHANISM OF O I LINES IN HERBIG AE/BE STARS
}

\author{
BLesson Mathew ${ }^{1}$ \\ Department of Physics, Christ University \\ Hosur Road, Bangalore 560029, India \\ and \\ Department of Astronomy and Astrophysics \\ Tata Institute of Fundamental Research \\ Homi Bhabha Road, Colaba, Mumbai 400005, India \\ P. MANOJ \\ Department of Astronomy and Astrophysics \\ Tata Institute of Fundamental Research \\ Homi Bhabha Road, Colaba, Mumbai 400005, India \\ MAYANK NARANG \\ Department of Astronomy and Astrophysics \\ Tata Institute of Fundamental Research \\ Homi Bhabha Road, Colaba, Mumbai 400005, India

\section{P. K. BANERJEE} \\ Astronomy and Astrophysics Division \\ Physical Research Laboratory \\ Navrangapura, Ahmedabad 380 009, India \\ PratheEKSha NAYAK \\ Indian Institute of Space Science and Technology (IIST) \\ Trivandrum, India

\section{S. MunEeR} \\ Indian Institute of Astrophysics \\ Koramangala, Bangalore 560034, India
}

S. VIG

Indian Institute of Space Science and Technology

Trivandrum, India

Pramod Kumar S.

Indian Institute of Astrophysics

Koramangala, Bangalore 560034, India

PAUL K. T.

Department of Physics, Christ University

Hosur Road, Bangalore 560029, India 


\author{
G. MAHESWAR \\ Indian Institute of Astrophysics \\ Koramangala, Bangalore 560034, India
}

${ }^{1}$ blesson.mathew@christuniversity.in

\title{
ABSTRACT
}

We have investigated the role of a few prominent excitation mechanisms viz. collisional excitation, recombination, continuum fluorescence and Lyman beta fluorescence on the O I line spectra in Herbig $\mathrm{Ae} / \mathrm{Be}$ stars. The aim is to understand which of them is the central mechanism that explains the observed $\mathrm{O}$ i line strengths. The study is based on an analysis of the observed optical spectra of 62 Herbig Ae/Be stars and near-infrared spectra of 17 Herbig Ae/Be stars. The strong correlation observed between the line fluxes of $\mathrm{O}$ I $\lambda 8446$ and $\mathrm{O}$ I $\lambda 11287$, as well as a high positive correlation between the line strengths of $\mathrm{O}$ I $\lambda 8446$ and $\mathrm{H} \alpha$ suggest that Lyman beta fluorescence is the dominant excitation mechanism for the formation of $\mathrm{O}$ I emission lines in Herbig Ae/Be stars. Further, from an analysis of the emission line fluxes of $\mathrm{O}$ I $\lambda \lambda 7774,8446$, and comparing the line ratios with those predicted by theoretical models, we assessed the contribution of collisional excitation in the formation of O I emission lines.

Keywords: stars pre-main sequence - stars: variables: T Tauri, Herbig Ae/Be (stars:) circumstellar matter - infrared: stars

\section{INTRODUCTION}

Herbig $\mathrm{Ae} / \mathrm{Be}(\mathrm{HAeBe})$ stars are intermediate mass $\left(2 M_{\odot} \leq M \leq 8 M_{\odot}\right)$ pre-main sequence stars with accretion disks, the innermost regions of which also act as a reservoir for the production of major emission lines seen in the optical and infrared spectra (Herbig 1960; Hillenbrand et al. 1992; Waters \& Waelkens 1998). HAeBe stars were first discussed as a distinct group of objects by Herbig (1960), who noted that they were stars of spectral type A or $\mathrm{B}$ with emission lines, located in an obscured region and often accompanied by a surrounding nebulosity. The present working definition of HAeBe stars includes, (a) pre-main sequence stars of $\mathrm{A}-\mathrm{F}$ spectral type, displaying emission lines in their spectra and (b) show a significant IR excess due to hot or cool circumstellar dust shell or a combination of both (The et al. 1994; Waters \& Waelkens 1998; Vieira et al. 2003). There have been extensive spectroscopic studies of HAeBe stars in the literature (e.g. Hamann \& Persson 1992; Hernandez et al. 2004; Manoj et al. 2006); particularly important are the recent studies by the X-Shooter team (Mendigutía et al. 2011, 2012; Fairlamb et al. 2015, 2017). Most of these studies have been devoted to $\mathrm{H} \alpha$ line analysis, the most prominent emission feature seen in the spectra of HAeBe stars (Finkenzeller \& Mundt 1984; Hamann \& Persson 1992). In the present study, we focus on the O I emission lines in the optical and near-infrared (1-2.5 micron) spectra in HAeBe stars.
O I $\lambda 8446$ is the most prominent O I emission line seen in the optical spectrum of HAeBe stars. This emission line results from the $3 s^{3} S^{0}-3 \mathrm{p}^{3} P$ transition and is seen as a triplet at high resolution, with wavelength values of $8446.25,8446.36$ and $8446.76 \AA$. It is present in the spectra of a wide variety of astrophysical sources such as planetary nebulae, novae and Seyfert galaxies. A number of studies have addressed the question of excitation mechanisms of OI emission lines in various astrophysical objects. Prominent mechanisms discussed for the formation of OI lines are collisional excitation, recombination, continuum fluorescence and Lyman beta $(\operatorname{Ly} \beta)$ fluorescence. For example, Grandi (1975b) showed that starlight continuum fluorescence is the favored excitation mechanism for the OI line in the Orion nebula whereas in Seyfert 1 galaxies it is excited by Lyman $\beta$ fluorescence (Grandi 1980). In novae, Strittmatter et al. (1977) identify Lyman $\beta$ fluorescence as the dominant excitation mechanism; a conclusion that has been supported by studies of several other novae (e.g Ashok et al. 2006; Banerjee \& Ashok 2012, and references therein). Ly $\beta$ fluorescence is identified as the dominant contributor to the emission strength of O I $\lambda 8446$ line in classical Be (hereafter CBe) stars, whether it is isolated (Slettebak 1951; Mathew et al. 2012b) or part of an X-ray binary system (Mathew et al. 2012a). Bhatia \& Kastner (1995) and Kastner \& Bhatia (1995) provided a theoretical framework of $\mathrm{O}$ I excitation and derived the 
expected line ratios of the prominent O I lines, when collisional excitation and $\operatorname{Ly} \beta$ fluorescence (referred as photoexcitation by accidental resonance (PAR process) in Bhatia \& Kastner 1995) are the dominant excitation mechanisms. From a comparative analysis of the theoretical estimates with the observed emission strengths of O I $\lambda \lambda 7774,8446,11287$ and 13165, Mathew et al. (2012b) demonstrated that Ly $\beta$ fluorescence is the dominant excitation mechanism for the production of $\mathrm{O}$ I $\lambda \lambda$ 8446, 11287 lines in CBe stars. CBe stars share similar spectral characteristics with HAeBe stars, such as emission lines of $\mathrm{H} \alpha$, OI, Fe II and Ca II triplet. It is worth exploring whether both $\mathrm{CBe}$ and $\mathrm{HAeBe}$ stars share similar excitation mechanism for the formation of O I lines. There could be considerable difference between the $\mathrm{O}$ I line forming regions in both the stellar systems. CBe stars are found to have a circumstellar gaseous decretion disk wherein O I $\lambda 8446$ line is formed at a mean radial distance of $\sim 8 R_{\star}$, considering Keplerian motion (Mathew et al. 2012b). However, the location of the origin of O I $\lambda 8446$ line in HAeBe is far from clear. Most of the accretion related emission lines in HAeBe stars (e.g. $\mathrm{H} \alpha, \mathrm{Pa} \beta, \mathrm{Br} \gamma)$ are thought to be formed in the magnetospheric accretion columns (Muzerolle et al. 2004). This work is an attempt to bring more clarity to our understanding of the formation mechanisms of $\mathrm{O}$ I emission lines in HAeBe stars.

The paper is organized as follows. In Section 2 we present the optical and near-infrared (near-IR) spectroscopic observations carried out over a period of 3 years and describe the data reduction techniques employed. We describe the methods and the python routines used for the spectral analysis and to estimate line flux in Section 3. The dominant excitation mechanism for the formation of O I lines in HAeBe stars is evaluated in Section 4. The main results of the paper are summarized in Section 5.

\section{OBSERVATIONS AND DATA REDUCTION}

The optical spectroscopic observations were carried out using the Himalayan Faint Object Spectrograph Camera (HFOSC) mounted on the 2-m Himalayan Chandra Telescope $(\mathrm{HCT})^{1}$. The spectroscopic observations were obtained with Grism 8 in combination with $167 l$ slit (1.92" wide and $11^{\prime}$ long), providing an effective resolving power of $\sim 1050$. The spectral coverage is from 5500 to $9000 \AA$, which included the spectral lines relevant to this study, viz., $\mathrm{H} \alpha$, O I $\lambda 7774$ and O I $\lambda 8446$. After each on-source exposure, FeNe lamp spectra were obtained for wavelength calibration. We have followed the regular procedure of reducing the spectra after bias

${ }^{1}$ http://www.iiap.res.in/iao/hfosc.html subtraction and flat-field correction using the standard tasks in Image Reduction and Analysis Facility (IRAF) ${ }^{2}$.

Near-IR spectra were obtained using the TIFR Near Infrared Spectrometer and Imager (TIRSPEC), mounted on the HCT. The spectra were obtained in $Y$ and $J$ passbands, at a resolving power of 1200 . The observations were performed in the dithered mode. Argon lamp spectra taken after each on-source exposure is used for wavelength calibration. An appropriate telluric standard (of early A spectral type) is observed at nearby airmass to the target object. The spectra of the target and the standard are reduced in a standard manner with the tasks in IRAF. For telluric correction, we removed the hydrogen lines from the telluric standard spectrum, which is then used to divide the object spectrum. The resultant object spectrum is multiplied with the blackbody corresponding to the spectral type of the telluric standard in order to preserve the continuum of the target spectrum. The log of optical and infrared spectroscopic observations is given in Table 1.

The sample of HAeBe stars observed were drawn from a larger list of $142 \mathrm{HAeBe}$ stars that we compiled from literature (The et al. 1994; Manoj et al. 2006; Fairlamb et al. 2015). Given the location of the observatory and the limiting magnitude of the spectrographtelescope combination, we were able to obtain the optical spectra of 56 HAeBe stars and near-IR spectra of 19 HAeBe stars. The observations were carried out over a period of 3 years, from 2014 to 2017. To increase the sample size of the present study we have included the optical spectra of HAeBe stars from Manoj et al. (2006), which were observed with similar observation setup. Thus we have optical spectra for a total of 62 HAeBe stars and near-IR spectra for 19 HAeBe stars. As a representative sample, we show $\mathrm{H} \alpha$, O I $\lambda \lambda 7774$, 8446 line profiles of V594 Cas, $\mathrm{LkH} \alpha 233$ and MWC 297 in Figure 1.

The $B, V, R_{C}$ magnitudes, total extinction $\left(A_{V}\right)$, spectral type and effective temperature $\left(T_{\text {eff }}\right)$ of 62 HAeBe stars are listed in Table 2. The spectral type is converted to $T_{\text {eff }}$ using the tabulated information in Pecaut \& Mamajek (2013). We compiled the photometric data from various sources in literature, whose references are given in Table 2. For some of the sources $R$ magnitudes are in Johnson system, which are converted to the Cousins system following Bessell (1983). The color excess, $E(B-V)$, is calculated from the observed $(B-V)$ colors and the intrinsic colors corre-

\footnotetext{
${ }^{2}$ IRAF is distributed by the National Optical Astronomy Observatories, which are operated by the Association of Universities for Research in Astronomy, Inc., under cooperative agreement with the National Science Foundation
} 

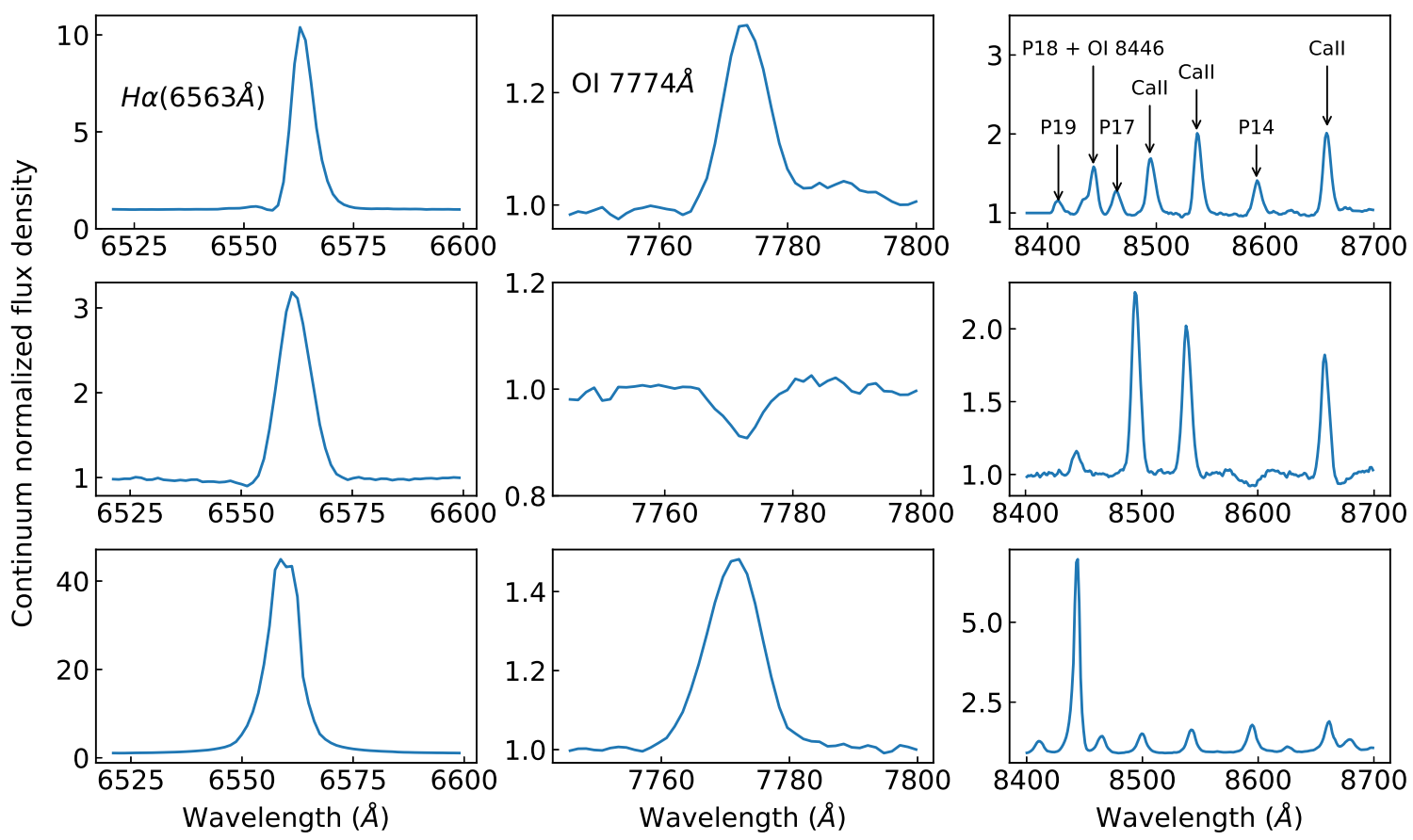

Figure 1. Observed spectra of V594 Cas, LkH $\alpha 233$ and MWC 297 (top to bottom). The line profiles of H $\alpha$, O I $\lambda 7774$ and O I $\lambda 8446$ are shown in each case (left to right). Ca II triplet $(8498,8542,8662 \AA)$ lines are seen in most spectra and appears to be blended with Paschen lines when they are in emission.

sponding to each spectral type, from the table listed in Pecaut \& Mamajek (2013). Further, we calculated $A_{V}$ from $E(B-V)$ considering a total-to-selective extinction value, $R_{V}=5$. It has been demonstrated from various studies (c.f. Hernandez et al. 2004) that $R_{V}=$ 5 is the preferred value in the analysis of HAeBe stars, suggesting grain growth in the disk of HAeBe stars (e.g. Gorti \& Bhatt 1993; Manoj et al. 2006)).

\section{ANALYSIS}

\subsection{Classification based on $\mathrm{O}_{\mathrm{I}}$ line profiles}

From the observed spectra, we find that $\mathrm{O}$ I lines, both in optical and infrared, are seen in emission as well as in absorption. We adopted the classification scheme proposed by Felenbok et al. (1988), wherein Group I stars have both O I $\lambda 8446$ and O I $\lambda 7774$ in emission; Group II sources are those with both lines in absorption; Group III is the case when O I $\lambda 8446$ is in emission and O I $\lambda 7774$ in absorption. We found 23 stars belonging to Group I, 16 in Group II and 23 in Group III classes. Similar classification scheme is applied to the infrared spectra. Although O I emission is evident among Group I stars, after subtracting the photospheric component, a net emission is seen in some of the Group II and Group III stars. For the current sample, we found net emission in O I $\lambda \lambda 7774,8446$ for 31 and 54 stars, respectively whereas 17 sources show net emission in O I $\lambda \lambda 11287$ and 13165 .

Table 1. Log of spectroscopic observations

\begin{tabular}{|c|c|c|c|c|c|}
\hline $\begin{array}{l}\text { Object } \\
\text { (1) }\end{array}$ & $\begin{array}{c}\text { Date of } \\
\text { Optical Observations } \\
(2)\end{array}$ & $\begin{array}{c}\text { Optical } \\
\text { Exp.time (s) } \\
(3)\end{array}$ & $\begin{array}{c}\text { Date of } \\
\text { IR Observations } \\
(4)\end{array}$ & $\begin{array}{c}Y \text { band } \\
\text { Exp.time }(\mathrm{s}) \\
(5)\end{array}$ & $\begin{array}{c}J \text { band } \\
\text { Exp.time (s) } \\
(6)\end{array}$ \\
\hline $51 \mathrm{Oph}$ & 2014 May. 19 & 60 & - & - & - \\
\hline AB Aur & 2017 Jan. 21 & 40 & 2013 Dec. 11 & 600 & 600 \\
\hline AS 442 & 2016 Aug. 10 & 300 & - & - & - \\
\hline AS 443 & 2016 Aug. 10 & 420 & - & - & - \\
\hline
\end{tabular}


Table 1 (continued)

\begin{tabular}{|c|c|c|c|c|c|}
\hline $\begin{array}{l}\text { Object } \\
\text { (1) }\end{array}$ & $\begin{array}{c}\text { Date of } \\
\text { Optical Observations } \\
(2)\end{array}$ & $\begin{array}{c}\text { Optical } \\
\text { Exp.time (s) } \\
(3)\end{array}$ & $\begin{array}{c}\text { Date of } \\
\text { IR Observations } \\
(4)\end{array}$ & $\begin{array}{c}Y \text { band } \\
\text { Exp.time (s) } \\
(5)\end{array}$ & $\begin{array}{c}J \text { band } \\
\text { Exp.time (s) } \\
(6)\end{array}$ \\
\hline AS 505 & 2016 Aug. 10 & 300 & 2016 Nov. 21 & 600 & 600 \\
\hline $\mathrm{BD}+30549$ & 2016 Nov. 22 & 400 & - & - & - \\
\hline $\mathrm{BD}+404124$ & 2014 Jun. 19 & 300 & - & - & - \\
\hline $\mathrm{BD}+651637$ & 2016 Aug. 10 & 300 & - & - & - \\
\hline CQ Tau & 2016 Nov. 23 & 300 & 2016 Nov. 21 & 600 & 600 \\
\hline HBC 334 & 2017 Jan. 03 & 1800 & - & - & - \\
\hline HBC 551 & 2014 Feb. 25 & 1200 & - & - & - \\
\hline HD 141569 & 2014 Jun. 19 & 300 & - & - & - \\
\hline \multirow[t]{2}{*}{ HD 142666} & 2014 Feb. 24 & 300 & - & - & - \\
\hline & 2014 Мay. 19 & 300 & - & - & - \\
\hline HD 144432 & 2014 May. 19 & 300 & - & - & - \\
\hline HD 145718 & 2016 Маy. 15 & 300 & - & - & - \\
\hline \multirow[t]{2}{*}{ HD 150193} & 2014 May. 19 & 600 & - & - & - \\
\hline & 2014 Jun. 19 & 300 & - & - & - \\
\hline HD 163296 & 2016 Мау. 15 & 30 & 2016 May. 15 & 120 & 120 \\
\hline HD 169142 & 2014 May. 19 & 300 & - & - & - \\
\hline HD 190073 & 2014 Oct. 02 & 60 & - & - & - \\
\hline \multirow[t]{2}{*}{ HD 200775} & 2017 Jan. 22 & 30 & 2016 Nov. 20 & 120 & 120 \\
\hline & - & - & 2017 Jan. 22 & 320 & 320 \\
\hline HD 216629 & 2016 Aug. 10 & 30 & - & - & - \\
\hline HD 245185 & 2017 Jan. 03 & 600 & - & - & - \\
\hline HD 250550 & 2017 Jan. 21 & 600 & 2017 Jan. 22 & 600 & 600 \\
\hline HD 259431 & 2015 Dec. 16 & 60 & 2016 Nov. 21 & 600 & 480 \\
\hline HD 31648 & 2016 Nov. 20 & 60 & 2016 Nov. 20 & 180 & 240 \\
\hline HD 35187 & 2017 Jan. 21 & 60 & - & - & - \\
\hline HD 35929 & 2017 Jan. 21 & 120 & 2016 Nov. 21 & 480 & 480 \\
\hline \multirow[t]{2}{*}{ HD 36112} & 2014 Feb. 24 & 180 & 2014 Feb. 24 & 120 & 120 \\
\hline & 2015 Jan. 27 & 90 & 2016 Nov. 21 & 360 & 360 \\
\hline HD 37490 & 2017 Jan. 21 & 20 & 2017 Jan. 21 & 320 & 400 \\
\hline HD 37806 & 2016 Nov. 22 & 30 & - & - & - \\
\hline HD 52721 & 2016 Nov. 22 & 30 & 2016 Nov. 21 & 180 & 180 \\
\hline HD 53367 & 2016 Nov. 23 & 90 & 2016 Nov. 21 & 180 & 180 \\
\hline HK Ori & 2017 Jan. 03 & 600 & - & - & - \\
\hline LkHa 167 & 2016 Sep. 25 & 1200 & - & - & - \\
\hline LkHa 198 & 2015 Dec. 16 & 1200 & - & - & - \\
\hline LkHa 224 & 2016 May. 16 & 900 & - & - & - \\
\hline LkHa 233 & 2014 Oct. 02 & 1800 & - & - & - \\
\hline LkHa 234 & 2016 Aug. 10 & 600 & - & - & - \\
\hline LkHa 257 & 2016 Aug. 10 & 900 & - & - & - \\
\hline MWC 1080 & 2014 Aug. 17 & 180 & 2014 Aug. 17 & 200 & 200 \\
\hline
\end{tabular}


Table 1 (continued)

\begin{tabular}{|c|c|c|c|c|c|}
\hline $\begin{array}{l}\text { Object } \\
(1) \\
\end{array}$ & $\begin{array}{c}\text { Date of } \\
\text { Optical Observations } \\
(2) \\
\end{array}$ & $\begin{array}{c}\text { Optical } \\
\text { Exp.time (s) } \\
(3) \\
\end{array}$ & $\begin{array}{c}\text { Date of } \\
\text { IR Observations } \\
(4) \\
\end{array}$ & $\begin{array}{c}Y \text { band } \\
\text { Exp.time (s) } \\
(5) \\
\end{array}$ & $\begin{array}{c}J \text { band } \\
\text { Exp.time (s) } \\
(6) \\
\end{array}$ \\
\hline \multirow{3}{*}{ MWC 297} & 2014 Nov. 02 & 180 & - & - & - \\
\hline & 2014 May. 19 & 360 & 2014 Jun. 19 & 120 & 80 \\
\hline & 2014 Jun. 19 & 600 & 2014 Aug. 17 & 200 & 120 \\
\hline PDS 174 & 2017 Jan. 03 & 900 & - & - & - \\
\hline PX Vul & 2014 Oct. 01 & 600 & - & - & - \\
\hline SV Cep & 2016 Aug. 10 & 300 & - & - & - \\
\hline UX Ori & 2017 Jan. 03 & 60 & - & - & - \\
\hline UY Ori & 2017 Jan. 03 & 900 & - & - & - \\
\hline V1012 Ori & 2017 Jan. 03 & 900 & - & - & - \\
\hline V1366 Ori & 2017 Jan. 03 & 600 & - & - & - \\
\hline V376 Cas & 2014 Oct. 02 & 1800 & - & - & - \\
\hline \multirow[t]{2}{*}{ V594 Cas } & 2014 Oct. 01 & 120 & 2016 Nov. 20 & 300 & 400 \\
\hline & 2014 Nov. 03 & 180 & - & - & - \\
\hline V699 Mon & 2016 Nov. 23 & 600 & - & - & - \\
\hline VV Ser & 2016 May. 15 & 1200 & - & - & - \\
\hline VY Mon & 2017 Jan. 22 & 900 & 2017 Jan. 22 & 500 & 600 \\
\hline \multirow[t]{2}{*}{ WW Vul } & 2016 Aug. 10 & 600 & - & - & - \\
\hline & 2016 May. 15 & 600 & - & - & - \\
\hline XY Per & 2016 Nov. 22 & 300 & 2017 Jan. 22 & 400 & 600 \\
\hline Z CMa & 2014 Feb. 24 & 180 & 2014 Feb. 24 & 120 & 40 \\
\hline
\end{tabular}

\subsection{Flux measurement of $O \mathrm{I}$ and $\mathrm{H \alpha}$ emission lines}

In this section we describe the method we used to measure the line fluxes of $\mathrm{H} \alpha$, O I $\lambda \lambda 7774,8446,11287$ and 13165 lines from the wavelength calibrated optical and near-IR spectra. The procedure can be summarized as, (i) estimating the equivalent width of the lines of interest from a Gaussian profile fit using LMFIT routine in Python, (ii) removing the contribution of Paschen P18 line from O I $\lambda 8446$, (iii) accounting for photospheric absorption using synthetic spectra, (iv) estimation of continuum flux at the wavelength region corresponding to $\mathrm{H} \alpha$ and $\mathrm{O}$ I lines, and (v) the calculation of extinction corrected line flux from the equivalent width and the continuum flux.

\subsubsection{Estimation of line equivalent width}

We estimated the equivalent width (EW) of O I $\lambda \lambda 7774,8446,11287,13165$ and $\mathrm{H} \alpha$ lines using the LMFIT module on the continuum subtracted, continuum normalized spectra. LMFIT, which is based on an Marquardt Levenberg nonlinear least squares minimization algorithm, was used to fit gaussians to the profiles.
3.2.2. Removal of Paschen line (P18) contribution from OI $\lambda 8446$

For the spectral resolution of our observations, the line profiles of O I $\lambda 8446$ and Paschen 18 (P18; $8437 \AA$ ) are blended (see Figure 1). We proposed a method in Mathew et al. (2012b) to deblend the P18 contribution from the net EW in the study of CBe stars, which will be employed here as well. The Paschen line strengths show a monotonic increase with wavelength and then display a trend of flattening out around P17 and beyond (Briot 1981). Hence it is reasonable to obtain the EW of P18 by linearly interpolating between the measured EW of P17 (8467 $\AA$ ) and P19 (8413 $\AA$ ) (see Mathew et al. $2012 b)$. This value is subtracted from the combined EW of O I $\lambda 8446$ and P18 to obtain the intrinsic EW of O I $\lambda 8446$.

\subsubsection{Accounting for Photospheric absorption}

The equivalent widths calculated from emission lines needs to be corrected for the photospheric absorption. The strength of the absorption component is estimated from the synthetic spectrum corresponding to the spec- 
tral type of the central star from Munari et al. (2005), which are calculated from the SYNTHE code (Kurucz 1993), using NOVER models as the input stellar atmospheres (Castelli et al. 1997). The EW of underlying absorption component for $\mathrm{H} \alpha$, O I $\lambda 7774$ and O I $\lambda 8446$ is estimated using the synthetic spectra corresponding to the spectral type of the star. Since the synthetic spectra of Munari et al. (2005) do not cover the infrared spectral region, we used NextGen (AGSS2009) theoretical spectra (Hauschildt et al. 1999) for the analysis of O I $\lambda \lambda 11287,13165$ line profiles. The equivalent width of the photospheric absorption is subtracted from the EW of the observed emission line to obtain the net equivalent width.
The equivalent width of $\mathrm{O}$ I emission lines, corrected for photospheric absorption, needs to be multiplied with the underlying stellar continuum flux density to obtain the line flux. We are taking the extinction corrected $R$-band flux density as a proxy for the continuum flux density underlying $\mathrm{H} \alpha$ line. The method of calculating the continuum flux density at $\mathrm{O}$ I emission lines from $\mathrm{H} \alpha$ line is described below. The continuum flux density at $\mathrm{H} \alpha$ is given as,

$$
F_{\nu, \text { cont }}(H \alpha)=F_{\nu, 0} \times 10^{\left(\frac{-R_{0}}{2.5}\right)}
$$

where $F_{\nu, 0}=3.08 \times 10^{-23} W m^{-2} H^{-1}$ and $\mathrm{R}_{0}$ is the extinction corrected $R_{C}$ magnitude. The extinction in $R$-band, $A_{R}$, is estimated from $\mathrm{A}_{V}$ using the extinction curve of McClure (2009).

Table 2. List of compiled stellar parameters for analysis of optical lines

\begin{tabular}{|c|c|c|c|c|c|c|c|c|}
\hline $\begin{array}{c}\text { Source } \\
(1) \\
\end{array}$ & $\begin{array}{c}\text { Sp. type } \\
(2)\end{array}$ & $\begin{array}{c}\text { Ref. Sp. type } \\
(3) \\
\end{array}$ & $\begin{array}{c}T_{\text {eff }}(\mathrm{K}) \\
(4)\end{array}$ & $\begin{array}{c}V \\
(5) \\
\end{array}$ & $\begin{array}{c}B-V \\
(6) \\
\end{array}$ & $\begin{array}{r}R_{C} \\
(7) \\
\end{array}$ & $\begin{array}{c}\text { Ref. Photometry } \\
(8) \\
\end{array}$ & $\begin{array}{l}A_{V} \\
(9) \\
\end{array}$ \\
\hline $51 \mathrm{Oph}$ & B9.5 IIIe & 1 & 10400 & 4.78 & 0.03 & 4.75 & 1 & 0.4 \\
\hline AB Aur & A1 & 1 & 9200 & 7.05 & 0.12 & 6.92 & 1 & 0.39 \\
\hline AS 442 & $\mathrm{~B} 8 \mathrm{Ve}$ & 14 & 12500 & 10.9 & 0.66 & 10.18 & 3 & 3.85 \\
\hline AS 443 & B2 & 1 & 20600 & 11.35 & 0.66 & 10.78 & 1 & 4.35 \\
\hline AS 505 & B5Vep & 15 & 15700 & 10.85 & 0.43 & 10.66 & 4 & 2.93 \\
\hline $\mathrm{BD}+30549$ & B8p & 16 & 12500 & 10.56 & 0.35 & 10.42 & 4 & 2.3 \\
\hline $\mathrm{BD}+404124$ & B3 & 1 & 17000 & 10.69 & 0.78 & 9.92 & 1 & 4.79 \\
\hline $\mathrm{BD}+463471$ & $\mathrm{~A} 0$ & 1 & 9700 & 10.13 & 0.4 & 9.8 & 1 & 2 \\
\hline $\mathrm{BD}+651637$ & $\mathrm{~B} 4$ & 1 & 16700 & 10.18 & 0.39 & 9.79 & 1 & 2.78 \\
\hline BO Cep & $\mathrm{F} 4$ & 1 & 6640 & 11.6 & 0.56 & 11.21 & 1 & 0.74 \\
\hline CQ Tau & F3 & 1 & 6720 & 10.26 & 0.79 & 9.72 & 1 & 2.01 \\
\hline HBC 334 & B3 & 1 & 17000 & 14.52 & 0.57 & 13.95 & 1 & 3.74 \\
\hline HBC 551 & B8 & 1 & 12500 & 11.81 & 0.26 & 11.54 & 1 & 1.85 \\
\hline HD 141569 & $\mathrm{~A} 0 \mathrm{Ve}$ & 1 & 9700 & 7.1 & 0.1 & 7.03 & 1 & 0.5 \\
\hline HD 142666 & $\mathrm{~A} 8 \mathrm{Ve}$ & 1 & 7500 & 8.67 & 0.5 & 8.34 & 1 & 1.25 \\
\hline HD 144432 & A9IVe & 1 & 7440 & 8.17 & 0.36 & 7.92 & 1 & 0.53 \\
\hline HD 145718 & $\mathrm{~A} 5 \mathrm{Ve}$ & 8 & 8080 & 9.1 & 0.52 & 8.79 & 2 & 1.8 \\
\hline HD 150193 & A2IVe & 1 & 8840 & 8.64 & 0.49 & 8.28 & 1 & 2.08 \\
\hline HD 163296 & A1Vep & 1 & 9200 & 6.88 & 0.09 & 6.82 & 1 & 0.24 \\
\hline HD 169142 & $\mathrm{~A} 5 \mathrm{Ve}$ & 1 & 8080 & 8.15 & 0.28 & 7.95 & 1 & 0.6 \\
\hline HD 179218 & A0IVe & 1 & 9700 & 7.39 & 0.08 & 7.33 & 1 & 0.4 \\
\hline HD 190073 & A2IVe & 1 & 8840 & 7.73 & 0.13 & 7.7 & 1 & 0.28 \\
\hline HD 200775 & B3 & 1 & 17000 & 7.37 & 0.41 & 7.01 & 1 & 2.94 \\
\hline HD 216629 & $\mathrm{~B} 3 \mathrm{IVe}+\mathrm{A} 3$ & 17 & 17000 & 9.32 & 0.45 & 9.11 & 4 & 3.14 \\
\hline HD 245185 & $\mathrm{~A} 1$ & 1 & 9200 & 9.94 & 0.1 & 9.88 & 1 & 0.29 \\
\hline HD 250550 & B9 & 1 & 10700 & 9.54 & 0.07 & 9.41 & 1 & 0.7 \\
\hline
\end{tabular}


B. Mathew et AL.

Table 2 (continued)

\begin{tabular}{|c|c|c|c|c|c|c|c|c|}
\hline $\begin{array}{c}\text { Source } \\
(1) \\
\end{array}$ & $\begin{array}{c}\text { Sp. type } \\
(2)\end{array}$ & $\begin{array}{c}\text { Ref. Sp. type } \\
(3) \\
\end{array}$ & $\begin{array}{c}T_{e f f}(\mathrm{~K}) \\
(4)\end{array}$ & $\begin{array}{l}V \\
(5) \\
\end{array}$ & $\begin{array}{c}B-V \\
(6) \\
\end{array}$ & $\begin{array}{r}R_{C} \\
(7) \\
\end{array}$ & $\begin{array}{c}\text { Ref. Photometry } \\
(8) \\
\end{array}$ & $\begin{array}{l}A_{V} \\
(9) \\
\end{array}$ \\
\hline HD 259431 & B6 & 1 & 14500 & 8.73 & 0.27 & 8.36 & 1 & 2.05 \\
\hline HD 31648 & $\mathrm{~A} 3 \mathrm{Ve}$ & 1 & 8550 & 7.7 & 0.2 & 7.59 & 1 & 0.55 \\
\hline HD 35187 & $\mathrm{~A} 2 \mathrm{e}+\mathrm{A} 7$ & 1 & 8840 & 8.17 & 0.22 & 76.4 & 1 & 0.73 \\
\hline HD 35929 & F2III & 1 & 6810 & 8.13 & 0.42 & 7.87 & 1 & 0.23 \\
\hline HD 36112 & A5IVe & 1 & 8080 & 8.34 & 0.26 & 8.16 & 1 & 0.5 \\
\hline HD 37490 & B2 & 5 & 20600 & 4.57 & -0.11 & 4.59 & 5 & 0.5 \\
\hline HD 37806 & $\mathrm{~A} 2 \mathrm{Vpe}$ & 1 & 8840 & 7.95 & 0.04 & 7.89 & 1 & -0.17 \\
\hline HD 38120 & B9 & 1 & 10700 & 9.01 & 0.06 & 8.93 & 1 & 0.65 \\
\hline HD 52721 & B1 & 5 & 26000 & 6.62 & 0.06 & 6.53 & 5 & 1.69 \\
\hline HD 53367 & B0IV/Ve & 9 & 31500 & 6.95 & 0.42 & 6.67 & 2 & 3.64 \\
\hline HK Ori & $\mathrm{A} 4+\mathrm{G} 1 \mathrm{~V}$ & 1 & 8270 & 11.71 & 0.56 & 11.2 & 1 & 2.1 \\
\hline IP Per & A6 & 1 & 8000 & 10.47 & 0.33 & 10.24 & 1 & 0.8 \\
\hline LkHa 167 & $\mathrm{~A} 2$ & 6 & 8840 & 15.06 & 1.42 & 14.32 & 4 & 6.73 \\
\hline LkHa 198 & B9 & 1 & 10700 & 14.18 & 0.95 & 13.31 & 1 & 5.1 \\
\hline LkHa 224 & F9 & 1 & 6040 & 14.07 & 1.44 & 12.98 & 1 & 4.44 \\
\hline LkHa 233 & $\mathrm{~A} 4$ & 1 & 8270 & 13.56 & 0.84 & 12.91 & 1 & 3.5 \\
\hline LkHa 234 & B7 & 1 & 14000 & 12.21 & 0.9 & 11.49 & 1 & 5.14 \\
\hline LkHa 257 & B5 & 7 & 15700 & 13 & 0.3 & 12.72 & 4 & 2.28 \\
\hline MWC 1080 & B0eq & 1 & 31500 & 11.52 & 1.34 & 10.39 & 1 & 8.24 \\
\hline MWC 297 & $\mathrm{~B} 1.5 \mathrm{Ve}$ & 10 & 24800 & 12.03 & 2.24 & 10.18 & 2 & 12.46 \\
\hline PDS 174 & B3e & 11 & 17000 & 12.84 & 0.81 & 12.18 & 2 & 4.94 \\
\hline PX Vul & F3 & 1 & 6720 & 11.54 & 0.83 & 11.12 & 1 & 2.21 \\
\hline R Cra & A0 & 1 & 9700 & 12.2 & 1.09 & 11.03 & 1 & 5.45 \\
\hline SV Cep & A0 & 1 & 9700 & 10.98 & 0.39 & 10.68 & 1 & 1.95 \\
\hline UX Ori & A3 & 1 & 8550 & 10.4 & 0.33 & 10.13 & 1 & 1.2 \\
\hline UY Ori & B9 & 12 & 10700 & 12.79 & 0.37 & 12.56 & 2 & 2.2 \\
\hline V1012 Ori & A3e & 13 & 8550 & 12.04 & 0.42 & 11.61 & 2 & 1.65 \\
\hline V1366 Ori & A0 & 1 & 9700 & 9.89 & 0.16 & 9.8 & 1 & 0.8 \\
\hline V376 Cas & B5e & 1 & 15700 & 15.55 & 1.13 & 14.59 & 1 & 6.43 \\
\hline V594 Cas & B8 & 1 & 12500 & 10.58 & 0.56 & 10.03 & 1 & 3.35 \\
\hline V699 Mon & B6 & 1 & 14500 & 10.54 & 0.54 & 10.06 & 1 & 3.4 \\
\hline VV Ser & B6 & 1 & 14500 & 11.92 & 0.93 & 11.11 & 1 & 5.35 \\
\hline VY Mon & B8 & 1 & 12500 & 13.47 & 1.55 & 12.19 & 1 & 8.3 \\
\hline WW Vul & A3 & 1 & 8550 & 10.74 & 0.44 & 10.45 & 1 & 1.75 \\
\hline XY Per & A5 & 1 & 8080 & 9.21 & 0.49 & 8.86 & 1 & 1.65 \\
\hline $\mathrm{Z} \mathrm{CMa}$ & B0 IIIe & 1 & 31500 & 9.47 & 1.27 & 8.63 & 1 & 7.89 \\
\hline
\end{tabular}

References. (1) Manoj et al. (2006); (2) Fairlamb et al. (2015); (3) Mendigutía et al. (2012) ; (4) Zacharias et al. (2004); (5)Hillenbrand et al. (1992); (6) Cohen \& Kuhi (1979); (7) Liu et al. (2011); (8) Carmona et al. (2010);

(9)Tjin A Djie et al. (2001); (10) Drew et al. (1997); (11) Gandolfi et al. (2008); (12) Vieira et al. (2003);

(13) Lee \& Chen (2007); (14) Mora et al. (2001); (15) Garrison (1970); (16) McDonald et al. (2017); (17) Skiff (2014);

The continuum flux densities of $\mathrm{O}$ I lines 7774 and 8446 are estimated from $\mathrm{H} \alpha$ continuum flux using the 
relation,

$$
\begin{aligned}
& F_{\lambda, \text { cont }}(7774)=\left(\frac{F_{\lambda c}(7774)}{F_{\lambda c}(H \alpha)}\right) \times F_{\lambda, \text { cont }}(H \alpha) \\
& F_{\lambda, \text { cont }}(8446)=\left(\frac{F_{\lambda c}(8446)}{F_{\lambda c}(H \alpha)}\right) \times F_{\lambda, \text { cont }}(H \alpha)
\end{aligned}
$$

The ratio of continuum flux densities, $\frac{F_{\lambda c}(7774)}{F_{\lambda c}(H \alpha)}$ and $\frac{F_{\lambda c}(8446)}{F_{\lambda c}(H \alpha)}$ are calculated using the synthetic spectra given in Munari et al. (2005). The line fluxes of O I $\lambda 7774$ and O I $\lambda 8446$ are obtained by taking a product of the continuum flux density with the measured equivalent width. Similarly, the continuum flux density in the nearIR region is calculated from the extinction corrected $J$ magnitudes of HAeBe stars. Further, the flux values of O I $\lambda 11287 \&$ O I $\lambda 13165$ are calculated from the continuum flux densities and the measured equivalent widths.

\section{RESULTS \& DISCUSSION}

\subsection{Excitation mechanisms for $O \mathrm{I}$ emission}

The excitation mechanisms contributing to O I emission that are discussed extensively in literature are recombination, collisional excitation, continuum fluorescence and Lyman $\beta$ fluorescence (Grandi 1975b; Strittmatter et al. 1977; Grandi 1980; Ashok et al. 2006; Banerjee \& Ashok 2012). In this section, we assess which one of the above is the dominant mechanism for the production of O I lines in HAeBe stars.

\subsubsection{Recombination}

One of the possible formation mechanism of permitted O I emission lines is through recombination followed by cascade from higher ionization states. However, recombination process alone is not sufficient to explain the strength of $\mathrm{O}$ I lines in systems such as Orion nebula (Grandi 1975b).

If the recombination process is the dominant mechanism, then the emission strengths of $\lambda 7774$ and $\lambda 8446$ should follow the ratio of statistical weights, i.e., $\mathrm{F}(\lambda 7774) / \mathrm{F}(\lambda 8446)=5 / 3$ (Strittmatter et al. 1977; Grandi 1980). So, if recombination operates in HAeBe stars, we should expect O I $\lambda 7774$ to be stronger than O I $\lambda 8446$. The flux ratio of $\mathrm{O}$ I $\lambda 7774$ and $\lambda 8446$ is shown as a function of $\mathrm{F}(\lambda 8446)$ in Figure 2. For $77 \%$ of HAeBe stars, the emission strength of O I $\lambda 8446$ is stronger than $\mathrm{O}$ I $\lambda 7774$. Hence, recombination is not likely to be the dominant excitation mechanism for the production of $\mathrm{O}$ I lines in $\mathrm{HAeBe}$ stars.

\subsubsection{Collisional Excitation}

Bhatia \& Kastner (1995) built a hybrid model to compute the collisionally excited level populations and line

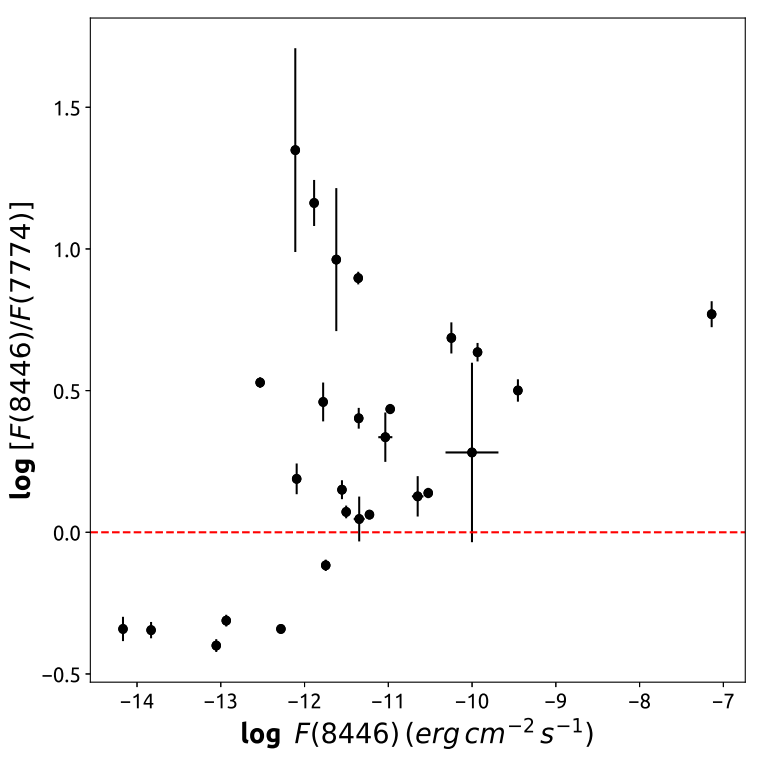

Figure 2. Log-Log plot of $\mathrm{F}(\lambda 8446) / \mathrm{F}(\lambda 7774)$ vs $\mathrm{F}(\lambda 8446)$ : The error bars are indicated. In $77 \%$ cases, $\mathrm{F}(\lambda 8446)>$ $\mathrm{F}(\lambda 7774)$.

intensities of neutral oxygen under optically thin conditions. The intensities of all possible allowed and forbidden $\mathrm{O}$ I lines in ultraviolet, visible and infrared wavelength regions were calculated over a range of densities and temperatures seen in astrophysical systems. Kastner \& Bhatia (1995) estimated the expected values of $\frac{F(8446)}{F(7774)}$ for various temperature, density combinations for collisional excitation. In the magnetospheric accretion models for HAeBe stars (e.g. Muzerolle et al. 2004), most of the emission lines observed in the visible and near-IR wavelengths are formed in magnetospheric accretion columns. It is possible that $\mathrm{O}$ I lines also form in these accretion columns. The typical accretion rates for HAeBe stars are in the range of $1.0 \times 10^{-8}-1.0 \times 10^{-6}$ $\mathrm{M}_{\odot} \mathrm{yr}^{-1}$ with a median value of $\sim 2.0 \times 10^{-7} \mathrm{M}_{\odot} \mathrm{yr}^{-1}$ (e.g. Mendigutía et al. 2011, 2012). The corresponding density of accretion columns are in the range of $10^{11}$ $-10^{13} \mathrm{~cm}^{-3}$ for temperatures of $6000-10000 \mathrm{~K}$, for typical parameters of magnetospheric accretion models (see Muzerolle et al. 2004, 1998, 2001; Hartmann et al. 1994). We have taken theoretical O I line flux ratio values corresponding to these temperature, density combinations from Kastner \& Bhatia (1995). Observational data is shown in Figure 3 for 50 HAeBe stars, including the measurements of 30 sources from Fairlamb et al. (2017). The flux values of O I $\lambda 7774$ and $\lambda 8446$ corresponding to a temperature of $5000 \mathrm{~K}$ and densities of $10^{10}, 10^{11}, 10^{12} \mathrm{~cm}^{-3}$ are represented as dotted lines in Figure 3 and $T=10,000 \mathrm{~K}, \mathrm{n}_{e}=10^{10}, 10^{11}, 10^{12} \mathrm{~cm}^{-3}$ combinations are shown in dashed lines. Figure 3 shows that the observed flux ratio for almost all the sources in 
our sample is greater than those predicted for densities $>10^{11} \mathrm{~cm}^{-3}$. Additionally, models for $\mathrm{H} \alpha$ emission in HBe stars also require densities of $\mathrm{n}_{e}=2 \times 10^{12} \mathrm{~cm}^{-3}$ for the line forming region (see Patel et al. 2016, 2017). Although these studies do not discuss about O I line forming region, the strong correlation between $\mathrm{H} \alpha$ and O I line emission (see Section 4.1.4) indicates that both lines are formed in the same region. Thus, our analysis suggest that collisional excitation may not be the prominent mechanism at densities $>10^{11} \mathrm{~cm}^{-3}$ seen in the line forming regions of $\mathrm{HAeBe}$ stars.

We have included O I $\lambda \lambda 7774,8446$ line measurements of a sample of HAeBe stars studied in Fairlamb et al. (2017). These objects were observed with X-shooter spectrograph mounted at Very Large Telescope, Chile. Figure 3 shows that the inclusion of the sample of $\mathrm{HAeBe}$ stars from X-Shooter provides more data in the lower flux regime of 7774 and 8446 lines. Further, O I $\lambda 8446$ flux values are more intense than the theoretical estimates corresponding to $T=5000 / 10000 \mathrm{~K}$ and $\mathrm{n}_{e}=10^{11} \mathrm{~cm}^{-3}$. This analysis strengthens the claim that collisional excitation is not the dominant excitation mechanism for the production of $\mathrm{O}$ I emission lines in HAeBe stars.

Further confirmation is obtained from the analysis of the infrared spectra of HAeBe stars. It has been proposed that if collisional excitation is the dominant excitation mechanism, the equivalent width of $\lambda 13165$ should be greater than that of $\lambda 11287$ i.e., $W(13165) / W(11287) \geq 1$ at $\mathrm{T}=10,000$ and $20,000 \mathrm{~K}$, respectively, for $\mathrm{n}_{e}=10^{10}-10^{12} \mathrm{~cm}^{-3}$ (Bhatia \& Kastner 1995). For most of our sample of stars we found that the emission line strength of O I $\lambda 11287$ higher than that of $\lambda 13165$ (see Figure 4), confirming that collisional excitation does not play a major role in the formation of O I emission lines in HAeBe stars.

\subsubsection{Continuum fluorescence}

Continuum fluorescence was invoked as the excitation mechanism for the production of $\mathrm{O}$ I lines in the spectra of planetary nebulae (Seaton 1968) and Orion Nebula (Grandi 1975b). Grandi in his thesis (Grandi 1975a) and in a paper summarizing the thesis results (Grandi 1975b) showed that the expected theoretical ratio of the line strengths of the $\lambda 13165$ and $\lambda 11287$ lines due to starlight excitation (equivalently continuum fluorescence) should be of the order of 10 or slightly more. These model predictions are summarized in Tables 7 and Table 2 of Grandi (1975a) and Grandi (1975b) respectively and also described in the text. In essence, $\lambda 13165$ is predicted to be much stronger than $\lambda 11287$ if continuum fluorescence is the dominant excitation mechanism for the O I lines (also see Strittmatter et al. 1977).

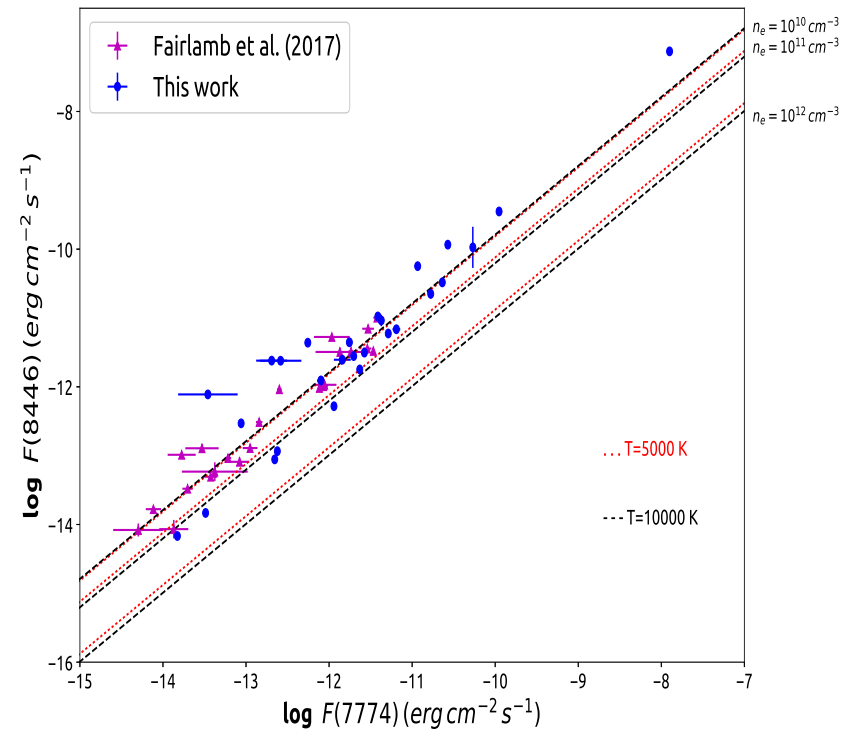

Figure 3. Log-Log plot of F(8446) vs. F(7774): red dotted lines correspond to theoretical flux values for $\mathrm{T}=5000 \mathrm{~K}$ and black dashed lines correspond to $\mathrm{T}=10000 \mathrm{~K}$, for $\mathrm{n}_{e}=$ $10^{10}, 10^{11}, 10^{12} \mathrm{~cm}^{-3}$ (Kastner \& Bhatia 1995). The sources from Fairlamb et al. (2017) are shown in purple triangles.

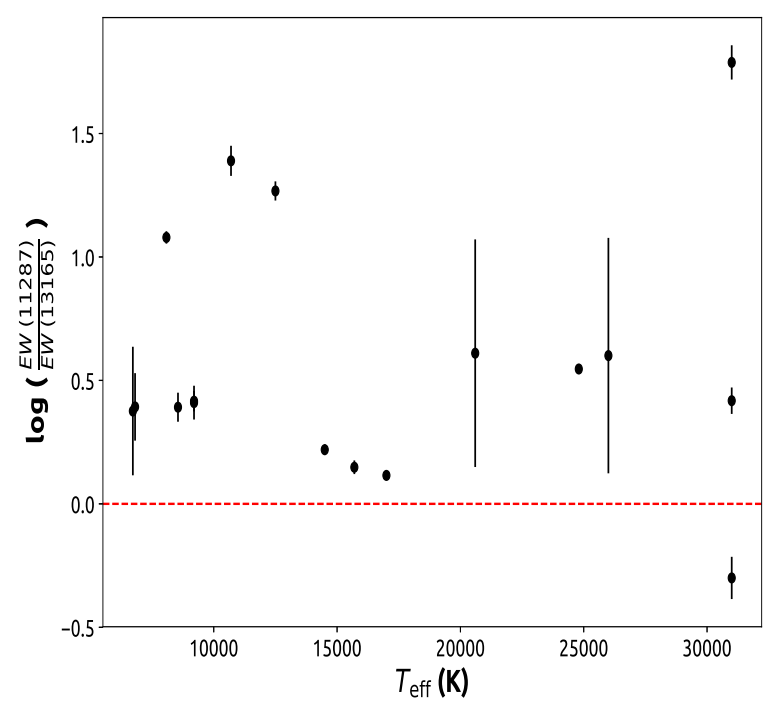

Figure 4. Plot of ratio of EWs of O I $\lambda 11287$ to O I $\lambda 13165$ against effective temperature of the star. It can be seen that in most cases $\frac{E W(11287)}{E W(13165)}>1$.

This prediction by Grandi was confirmed observationally for the Orion nebula in the spectroscopic studies by Lowe, Moorhead \& WehlauLowe (1977). Also, strong O I emission lines at $7002 \AA, 7254 \AA$ and $7990 \AA$ lines would be observed in the spectra (Strittmatter et al. 1977; Grandi 1980). Apart from the Orion nebula, another instance where the $\lambda 13165$ line is stronger than the $\lambda 11287$ line is in the inner 10 arcsecond sized neb- 
ula surrounding P Cygni. Near-infrared 1-2.5 micron spectra by Smith \& Hartigan (2006) of this region gives a value of $2.55 \pm 0.57$ for the ratio of the $13165 \& 11287$ line strengths.

Our analysis show that the emission strength of O I $\lambda 11287$ is greater than that of O I $\lambda 13165$ for our sample of HAeBe stars (Figure 4). In addition, we do not see emission lines at $7002 \AA, 7254 \AA$ and $7990 \AA$ in any of the object spectra. This suggests that continuum fluorescence is unlikely to be the dominant mechanism for the formation of $\mathrm{O}$ I emission lines in HAeBe stars.

\subsubsection{Lyman $\beta$ fluorescence}

Lyman $\beta$ (Ly $\beta$ ) fluorescence occurs because the $3 d^{3} D^{0}$ level of $\mathrm{O}$ I is populated by $\mathrm{Ly} \beta$ radiation, with subsequent cascades producing the O I $\lambda \lambda 11287,8446,1304$ lines in emission (Figure 5). This is due to the near coincidence in wavelength of Lyman beta and the O I resonance line $2 p^{3} P_{2}-3 d^{3} D_{321}^{0}$ at $1025.77 \AA$ (Bowen 1947). Our analysis show that the cascade lines expected from Ly $\beta$ fluorescence, O I $\lambda 8446$ and O I $\lambda 11287$ are quite strong in the spectra of HAeBe stars. Also, O I $\lambda 7774$ is less intense than O I $\lambda 8446$, suggesting that collisional excitation and recombination are relatively less important for O I excitation in HAeBe stars. Similarly, the lower emission strength of $\mathrm{O}$ I $\lambda 13165$ with respect to $\lambda 11287$ rules out collisional excitation and continuum fluorescence as the dominant mechanisms for the production of O I lines. Further, O I $\lambda \lambda 7002,7254,7990$ emission lines are not present in the spectra of HAeBe stars. These lines are generally seen in sources where O I lines are excited by continuum fluorescence. All these pieces of evidence strongly suggest that $\operatorname{Ly} \beta$ fluorescence is likely to be the dominant excitation mechanism for the production of $\mathrm{O}_{\mathrm{I}}$ lines in HAeBe stars.

If $\mathrm{Ly} \beta$ fluorescence is responsible for $\mathrm{O}$ I emission in HAeBe stars, then one would expect a correlation between $\mathrm{H} \alpha$ and $\mathrm{O}$ I $\lambda 8446$ line intensities. Ly $\beta$ photon results from the $\mathrm{n}=3-1$ transition of the hydrogen atom and the $\mathrm{H} \alpha$ photon results from the transition $\mathrm{n}$ $=3-2$. Thus the upper level of both the transitions are the same. In other words, hydrogen atoms in the excited state of $\mathrm{n}=3$ are responsible for both lines. If these lines originate from the same gas component, one would expect their intensities to be correlated. If, in addition, O I $\lambda 8446$ intensity is proportional to $\mathrm{Ly} \beta$ intensity, then one would expect a correlation between $\mathrm{H} \alpha$ and $\mathrm{O} \mathrm{I} \lambda 8446$. This is shown in Figure 6, where $\mathrm{F}(\lambda 8446)$ is shown as a function of $\mathrm{F}(\mathrm{H} \alpha)$ for our sample and those from Fairlamb et al. (2017). A correlation in seen between the flux values of $\mathrm{H} \alpha$ and $\mathrm{O}$ I $\lambda 8446$, suggesting the application of $\operatorname{Ly} \beta$ fluorescence process. A linear fit to the distribution of points in Figure 6 gives a relation of the form $\mathrm{F}(\lambda 8446) \propto \mathrm{F}(\mathrm{H} \alpha)^{1.02 \pm 0.04}$,

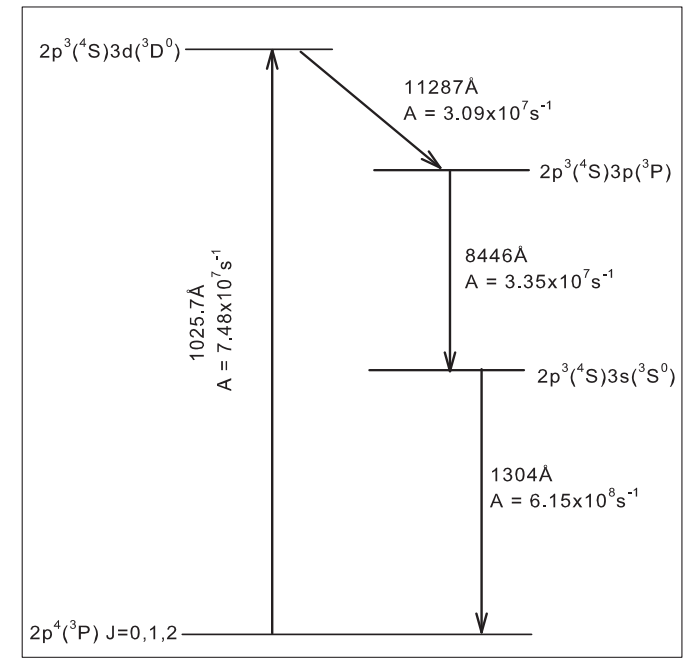

Figure 5. Figure shows the pumping and the fluorescent transitions in O I caused by the Lyman beta fluorescence process.

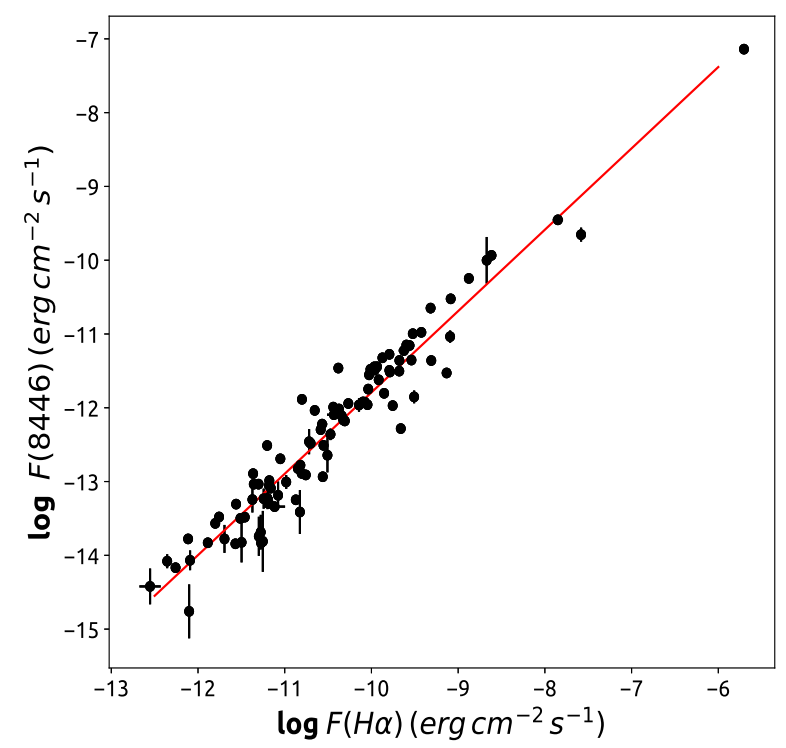

Figure 6. Log-Log plot of $\mathrm{F}(8446)$ vs $\mathrm{F}(\mathrm{H} \alpha)$ : The sample of HAeBe stars from our studies and from Fairlamb et al. (2017) are shown in black circles. We plotted 97 sources including 57 from Fairlamb et al. (2017), of which 7 are common between Fairlamb et al. (2017) and our sample.

with a Pearson's correlation coefficient of 0.96. From the analysis it is clear that $\mathrm{H} \alpha$ emission is correlated with the emission strength of O I $\lambda 8446$. This particularly has implications in understanding the region of formation of O I emission lines in HAeBe stars.

If $\mathrm{Ly} \beta$ photons and $\mathrm{O}$ I atoms do not co-exist, $\mathrm{Ly} \beta$ fluorescence would not have been possible since Ly $\beta$ gets scattered off neutral hydrogen resulting in the production of $\mathrm{Ly} \alpha$ and $\mathrm{H} \alpha$, before interacting with neutral oxy- 
gen atoms. This is the reason why $\operatorname{Ly} \beta$ fluorescence does not operate in Orion nebula where $\operatorname{Ly} \beta$ photons are trapped in the inner regions of the nebula whereas the O I is confined in the exterior (Grandi 1975b). In the case of HAeBe stars, $\mathrm{H} \alpha$ is thought to originate in the magnetospheric accretion columns which connects the inner disk to the central star. The fact that $\operatorname{Ly} \beta$ fluorescence operates in $\mathrm{HAeBe}$ stars suggests that $\mathrm{O}$ I emission lines are also formed in these accretion columns in HAeBe stars.

It is worth noting that the gas has to be optically thick in $\mathrm{H} \alpha$ in order for O I $\lambda 8446$ to get excited by Ly $\beta$ fluorescence. From the equations of level populations in statistical equilibrium, Grandi (1980) derived an optical depth in $\mathrm{H} \alpha\left(\tau_{H \alpha}\right)$ of 2000 , considering $\mathrm{Ly} \beta$ fluorescence operating in active galaxies. We examined whether the line forming region in HAeBe stars are optically thick in $\mathrm{H} \alpha$. For the sample of HAeBe stars considered in this study, the median flux ratio $\mathrm{F}(\mathrm{H} \alpha / \lambda 8446)_{\text {obs }}$ is 65.2 . From the analysis of level populations, Strittmatter et al. (1977) derived a theoretical $\mathrm{H} \alpha$ to $\mathrm{O}$ I $\lambda 8446$ flux ratio of around 7500 , under optically thin conditions. The optical depth of $\mathrm{H} \alpha$ can be estimated from the ratio of theoretical to observed line flux ratio, i.e., $\tau_{H \alpha}=7500 / \mathrm{F}(\mathrm{H} \alpha / \lambda 8446)_{o b s}=$ 115. The sufficiently high value of optical depth in $\mathrm{H} \alpha$ derived in the case of HAeBe stars agrees with the assumption that the gas needs to be optically thick thereby trapping the Lyman beta photons, paving way for $\mathrm{Ly} \beta$ fluorescence.

\section{CONCLUSION}

From an analysis of the observed optical spectra of $62 \mathrm{HAeBe}$ stars and near-infrared spectra of $17 \mathrm{HAeBe}$ stars, we have shown that $\operatorname{Ly} \beta$ fluorescence is likely to be the dominant excitation mechanism for the formation of $\mathrm{O}$ I emission lines. We ruled out recombination and continuum fluorescence as the possible excitation mechanisms as the emission strength of $\mathrm{O}$ I $\lambda 8446$ and $\lambda 11287$ are much stronger than the adjacent $\mathrm{O}$ I lines at $\lambda 7774$ and $\lambda 13165$, respectively. We found that collisional excitation does not contribute substantially to O I emission from the comparative analysis of the observed line flux values of $\lambda 7774$ and $\lambda 8446$ with those predicted by the theoretical models of Kastner \& Bhatia (1995).

\section{ACKNOWLEDGMENTS}

We would like to thank the referee for their comments which helped in improving the quality of the manuscript. We would like to thank the staff at IAO, Hanle and its remote control station at CREST, Hosakote for their help during the observation runs. This research uses the SIMBAD astronomical data base service operated at CDS, Strasbourg. This publication made use data of 2MASS, which is a joint project of University of Massachusetts and the Infrared Processing and Analysis Centre/California Institute of Technology, funded by the National Aeronautics and Space Administration and the National Science Foundation.

\section{REFERENCES}

Ashok, N. M., Banerjee, D. P. K., Varricatt, W. P., Kamath, U. S. 2006, MNRAS, 368, 592

Banerjee, D. P. K., Ashok, N. M. 2012, BASI, 40, 243

Bessell, M. S. 1983, PASP, 95, 480

Bhatia, A. K., Kastner, S. O. 1995, ApJS, 96, 325

Bowen, I. S. 1947, PASP, 59, 196

Briot, D. 1981, A\&A, 103, 5

Cohen, M., Kuhi, L. V. 1979, ApJS, 41, 743

Calvet, N., Gullbring, E. 1998, ApJ, 509, 802

Carmona, A., van den Ancker, M. E., Audard, M., et al. 2010, A\&A, 517, A67

Castelli, F., Gratton, R. G., Kurucz, R. L. 1997, A\&A, 318, 841

Cauley, P. W. \& Johns-Krull, C. M. 2015, ApJ, 810, 5

Corcoran, M., Ray, T. P. 1998, A\&A, 331, 147

Dahm, S. E. 2008, AJ, 136, 521

Drew, J. E., Busfield, G., Hoare, M. G., et al. 1997,MNRAS, 286, 538

Fairlamb, J. R., Oudmaijer, R. D., Mendigutia, I. et al. 2017, MNRAS, 464, 4721

Fairlamb, J. R., Oudmaijer, R. D., Mendigutia, I. et al. 2015, MNRAS, 453, 976

Fang, M., van Boekel, R., Wang, W. et al. 2009, A\&A, 504, 461

Felenbok, P., Czarny, J., Catala, C., Praderie, F. 1988, A\&A, 201, 247

Ferland, G., Netzer, H. 1979, ApJ, 229, 274

Finkenzeller, U., Mundt, R. 1984, A\&AS, 55, 109
Gandolfi, D., Alcalá, J. M., Leccia, S. et al. 2008, ApJ, 687, 1303

Garrison, R. F. 1970, AJ, 75, 1001

Gorti, U., Bhatt, H. C. 1993, A\&A, 270, 426

Grandi, S. A. 1975a, Ph.D. thesis, Univ. Arizona

Grandi, S. A. 1975b, ApJ, 196, 465

Grandi, S. A. 1980, ApJ, 238, 10

Gullbring, E., Hartmann, L., Briceno, C., Calvet, N. 1998, ApJ, 492,323

Hamann, F., Persson, S. E. 1992, ApJS, 82, 247

Hartmann, L., Hewett, R., Calvet, N. 1994, ApJ, 426, 669

Hauschildt, P. H., Allard, F., Baron, E. 1999, ApJ, 512, 377

Herbig G. H., 1960, ApJS, 4, 337

Herczeg, G. J. \& Hillenbrand, L. A. 2008, ApJ, 681, 594

Hernández, J., Calvet, N., Briceño, C. et al. 2004, AJ, 127, 1682

Hillenbrand, L. A., Strom, S. E., Vrba, F. J., Keene, J. 1992, ApJ, 397, 613

Ingleby, L., Calvet, N., Herczeg, G. et al. 2013, ApJ, 767, 112

Kastner, S. O., \& Bhatia, A. K. 1995, ApJ, 439, 346

Kenyon, S. J., Hartmann, L. 1995, ApJS, 101, 117

Kurucz R. 1993, ATLAS9 Stellar Atmosphere Programs and 2 km/s grid, Kurucz CD-ROM No. 13. Smithsonian

Astrophysical Observatory, Cambridge, MA

Lee, H.-T., \& Chen, W. P. 2007, ApJ, 657, 884

Liu, T., Zhang, H. Wu, Y. et al. 2011, ApJ, 734, 22

Lowe, R. P., Moorhead, J. M., Wehlau, W. H. 1977, ApJ, 214, 712 
Manoj, P., Bhatt, H. C., Maheswar, G., Muneer, S. 2006, ApJ, 653,657

Mathew, B., Banerjee, D. P. K., Naik, S., Ashok, N. M., 2012a, MNRAS, 423, 2486

Mathew, B., Banerjee, D. P. K., Subramaniam, A., Ashok, N. M., 2012b, ApJ, 753, 13

McDonald, I., Zijlstra, A. A., \& Watson, R. A. 2017, VizieR Online Data Catalog, 747,

Mora, A., Merín, B., Solano, E., et al. 2001, A\&A, 378, 116

Mathis, J. S. 1990, ARA\&A, 28, 37

Mendigutía, I., Calvet, N., Montesinos, B. et al. 2011, A\&A, 535, A99

Mendigutía I., Mora A., Montesinos B. et al. 2012, A\&A, 543, A59

McClure, M. 2009, ApJ, 693, L81

Munari, U., Sordo, R., Castelli, F., \& Zwitter, T. 2005, A\&A, 442, 1127

Muzerolle, J., D’Alessio, P., Calvet, N., \& Hartmann, L. 2004, ApJ, 617, 406

Muzerolle, J., Calvet, N., Hartmann, L. 2001, ApJ, 550, 944

Muzerolle, J., Calvet, N., Hartmann, L. 1998, ApJ, 492, 743

Patel, P., Sigut, T. A. A., Landstreet, J. D. 2017, ApJ, 836, 214
Patel, P., Sigut, T. A. A., Landstreet, J. D. 2016, ApJ, 817, 29

Pecaut, M. J., Mamajek, E. E. 2013, ApJS, 208, 9

Porter, J. M., \& Rivinius, T. 2003, PASP, 115, 1153

Seaton, M. J. 1968, MNRAS, 139, 129

Shu, F., Najita, J., Ostriker, E. et al. 1994, ApJ, 429, 781

Slettebak, A. 1951, ApJ, 113, 436

Smith, N., Hartigan, P. 2006, ApJ, 638, 1045

Skiff, B. A. 2014, VizieR Online Data Catalog, 1,

Strittmatter, P. A., Woolf, N. J., Thompson, R. I. et al. 1977, ApJ, 216, 23

The, P. S., de Winter, D., Perez, M. R. 1994, A\&AS, 104, 315

Tjin A Djie, H. R. E., van den Ancker, M. E., Blondel, P. F. C. et al. 2001, MNRAS, 325, 1441

Vieira, S. L. A., Corradi, W. J. B., Alencar, S. H. P. et al. 2003, AJ, 126, 2971

Waters L. B. F. M., Waelkens, C., 1998, ARA\&A, 36, 233

Zhong, J., Lépine, S., Li, J. et al. 2015, Research in Astronomy and Astrophysics, 15, 1154

Zacharias, N., Monet, D. G., Levine, S. E. et al. 2004, Bulletin of the American Astronomical Society, 36, 48 